\title{
Design and Simulation of Opera Lighting and Projection Effects
}

\author{
Julie O'B. Dorsey \\ François $X$. Sillion \\ Donald P. Greenherg \\ Program of Computer Graphics \\ Cornell University \\ Ithaca, New York 14853
}

\begin{abstract}
A major problem challenging opera designers is the inability to coordinate lighting, projection systems, and set designs in the preliminary planning phase. New computer graphics techniques, which provide the set and lighting designer the opportunity to evaluate, test, and control opera designs prior to the construction of full scale systems are presented. These techniques--light source input, simulation of directional lighting, modeling of scenic projection systems, and full three-dimensional simulation-show the potential for the use of computer graphics in theater design.

The light source input component consists of a program for assigning light source attributes with a set of theater lighting icons. This module allows a designer to specify light source characteristics in a way familiar to the discipline and to make preliminary evaluations of the lighting conditions.

An extended progressive radiosity method is introduced to simulate the directional lighting characteristics which are specified by the input program.

A new projection approach is presented to simulate the optical effects of scenic projectors. In addition, a solution to the distortion problem produced by angular projections is described.

The above components are integrated to produce full threedimensional simulations of the global illumination effects in an opera scene.
\end{abstract}

CR Categories and Subject Descriptors: I.3.0 [Computer Graphics]: General; I.3.7 [Computer Graphics]: Three Dimensional Graphics and Realism; J.2 [Computer Applications]: Performing Arts.

General Terms: Algorithms

Additional Keywords and Phrases: opera and stage design, angular projection, simulation, radiosity, directional light sources, texture mapping.

\section{Introduction}

Opera stage design is an extremely difficult task as, in addition to the standard architectural and aesthetic considerations, a number of

Permission to copy without fee all or part of this material is granted provided that the copies are not made or distributed for direct commercial advantage, the ACM copyright notice and the title of the publication and its date appear, and notice is given that copying is by permission of the Association for Computing Machinery. To copy otherwise, or to republish, requires a fee and/or specitic permission. additional issues are present, such as dynamic and intricate lighting and sets, projected background scenery, changing focus of attention, manipulation of implied perspective, multiple viewing points, motion of performers, and synchronization with music. Stage and lighting designers, as well as conductors, rarely have the opportunity to evaluate these effects together. Consequently, stage set and lighting designs are currently developed separately-being combined only in the last step of the process.

Presently, the only feasible method available for combining a limited stage and lighting design is the construction of small scale models. While this process does give some insight into the visual impact of the final production, it is a laborious. costly, incomplete, and time-consuming endeavor. Furthermore, because of their small scale, these models are so inadequate for the evaluation of complex lighting effects that they are not commonly used. Thus, in practice. the stage and lighting designers will often work in isolation from each other. The bulk of the lighting designer's task, then. occurs at the last minute-after the sets are assembled and in place on the stage.

The primary objective of this paper is to provide the stage and lighting designer the opportunity to design and evaluate the lighting and projected scenery prior to the actual implementation. In particular, techniques for light source descriptions and specification, the simulation of directional lighting. and the modeling of scenic projection systems have been developed. In addition, a solution for the distortion problem in angular projections is introduced. The procedures have been combined to provide full three-dimensional simulations so that the proposed design strategy can be evaluated from any viewer position. The variables are the positions of the stage sets, the locations, orientation, spatial emittance, and color of the lights, the number of lights which are illuminated, the background projection systems and scenery, all within the given theater geometry.

Three famous opera houses have been selected to demonstrate the system: the Metropolitan Opera at Lincoln Center in New York City, La Scala in Milan, Italy, and the Staatsoper in Vienna, Austria. Due to space limitations, only the Metropolitan Opera is illustrated.

\section{Input for Lights}

In general, light sources have well-defined finite geometries that greatly affect the distribution of the light emitted from the source. There are three types of abstract emissive geometries: point sources (zero dimensional), linear sources (one dimensional), and area sources (two dimensional) [23]. The light sources used in opera production can be treated as point sources, since the lights are very small and are located at a significant distance from the stage.

Perhaps the most important characteristic of a luminaire that must be included in a complete model of a light source is the luminous 
intensity distribution. In contrast to the assumption typically used in computer graphics, most of the lights used in opera production do not emit light of constant intensity in all directions.

A non-uniform intensity distribution must be specified, which describes the variations of the emitted intensity with direction. The lighting industry uses goniometric diagrams to represent these vector-valued functions for easy interpretation [3]. These diagrams represent a planar slice through the vector field and thus plot the relative intensity as a function of angular direction (Figure 1). For

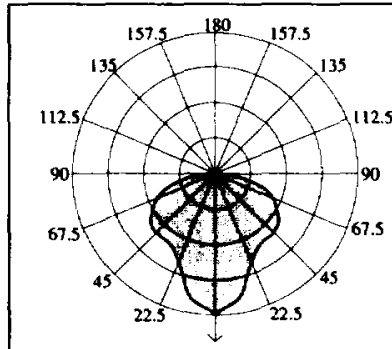

(a)

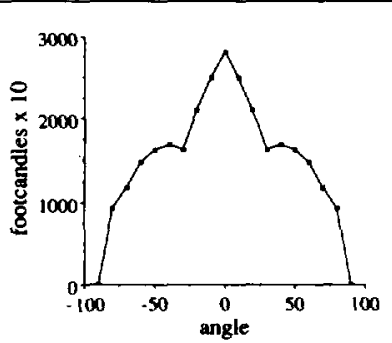

(b)
Figure 1: A sample emission distribution. (a) Polar goniometric diagram. (b) Corresponding cartesian diagram.

luminaires with concentrated beams, such as spotlights, cartesian coordinates are preferred because of the need for more precision than a polar curve allows.

\subsection{Instruments and Lamps}

In lighting design for opera, many different types of luminaires are used. Although there appear to be a large number of different instrument styles used, each style is a variation on five particular instruments: the ellipsoidal reflector spotlight; the Fresnel spotlight; the striplight; the ellipsoidal reflector floodlight; and the beam projector. The optical characteristics of each of these instruments (and variations thereof) have been modeled.

\subsection{Assigning Light Source Attributes}

An interactive graphical program has been developed to allow one to design a lighting scenario. While a final lighting layout is primarily a tool for communicating the designer's concept and intentions to the electricians, lighting crews, and board operators, who must "hang" the design and execute it in a performance, this program provides the means to develop ideas, experiment, move and change instruments and their attributes, and iteratively refine a design.

The input program allows for the complete specification of attributes for stage lighting. The user can specify the position of each instrument, its intensity pattern, color, projection pattern, and the area which it illuminates in addition to indicating its height above the floor and the angles of the beams of light. As the parameters associated with a light are adjusted, the lamp is instantly updated with the resulting beam and field angles as well as throw distance. This feature makes it possible to combine light sources and evaluate the design implications (e.g. if their beams overlap). Thus, while still in the modeling phase, one has a good idea of the overall lighting scheme. Once a preliminary design is specified, the user can simulate the illumination effects and, with a separate program, view the results to further refine the lighting design.

An attempt has been made to carefully design the graphical interface so the process of assigning attributes is similar to the way in which it is physically performed. A menu is available which contains a two dimensional graphical representation of the five major categories of lights used in opera production. Once a category has been selected, it is possible to choose from a variety of instrument types and manufacturers within that category.

Most of the luminaires used in theater production have a spot and flood focus intensity distribution associated with them. When a lamp has been selected, a two dimensional icon is drawn in one window, and the components of the lamp which move during the focusing of that particular instrument can be varied interactively, making it possible to focus the instrument to the desired setting (Figure 2). To specify the intensity distribution, one window shows either a cartesian or polar goniometric diagram of the current luminous intensity distribution or candle power distribution curve for the light source. This diagram is updated continuously as the instrument is focused. To scale the distribution, the maximum intensity which the lamp emits at the center of its beam is specified in units of candelas.

Each light source can have a unique pattern or slide. A library of patterns and slides to be used in projection has been compiled. The designer can select a pattern from the library and associate it with a given light source.

Color can be controlled by placing a transparent color filter between the light source and the receiving surface(s). Using the filter section of the input program, an interactive color tool allows the user to vary the characteristics of the filter used to color the light emitted by a lamp.

It is possible to position a lamp at any location relative to the stage environment. Most light sources are positioned on the light bridges, but occasionally they are placed on the front edge of the stage as footlights or on temporary ladder-like structures along the sides. To aid the user in positioning the lamps, one viewport displays the light source with three dimensional transparent cones attached to it (optionally displayed) in the model of the stage. These cones represent the beam and field angles as well as the throw distance of the instrument (see Appendix A). As the lights are positioned relative to the stage area, the cones allow the user to visualize the direction in which the light will be emitted as well as how much illumination a particular area will receive.

\section{Simulation of Theater Lighting Conditions}

Radiosity methods, derived from the field of radiative heat transfer, have been successfully applied to the area of realistic image synthesis $[4,5,6,9,16,24]$. The radiosity method has the attractive characteristic of providing a view-independent solution. Hence, once the solution has been performed, a hardware renderer can be used to display the scene from changing viewpoints at interactive rates.

\subsection{Modeling Directional Light Sources with Progres- sive Radiosity}

The progressive radiosity method [4] can be extended to account for directional variations in a light source with non-uniform emission distributions (Figure 1). In this implementation, the form-factors are computed using the ray-traced form-factor approach proposed by Wallace et al. [24]. To account for the variation in light source directionality, the form factor from the light source to a vertex is computed as usual and then weighted by a directionality scaling factor, $s$. Each directional light source has a distribution associated with it which describes its normalized light source intensity versus angle. The value of $s$ is obtained from this distribution for each element vertex based on the direction $\theta_{2}$ (the angle between the direction vector of the light source and the direction of an element vertex).

In this way, the amount of light which is transferred from the light source to the environment is weighted according to the directional 

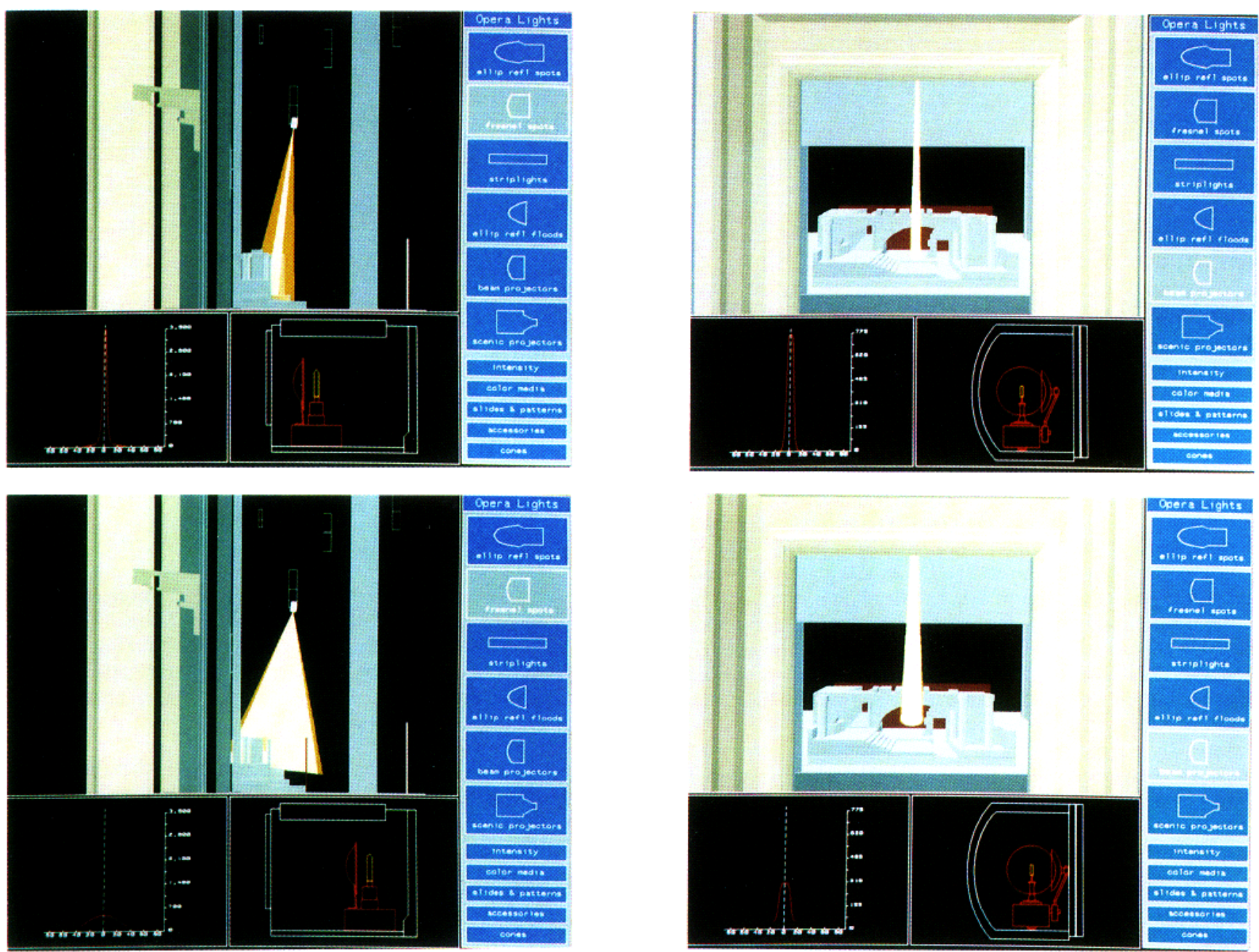

(a)

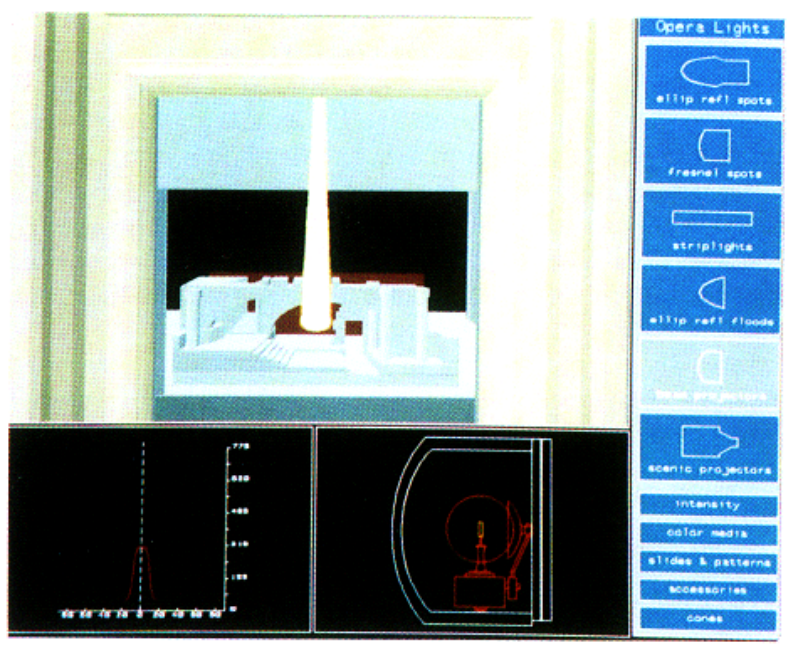

(b)

Figure 2: Light Source Input and Attribute Assignment. (a) The Fresnel spotlight - spot and flood foci. (b) The beam projector - spot and flood foci.

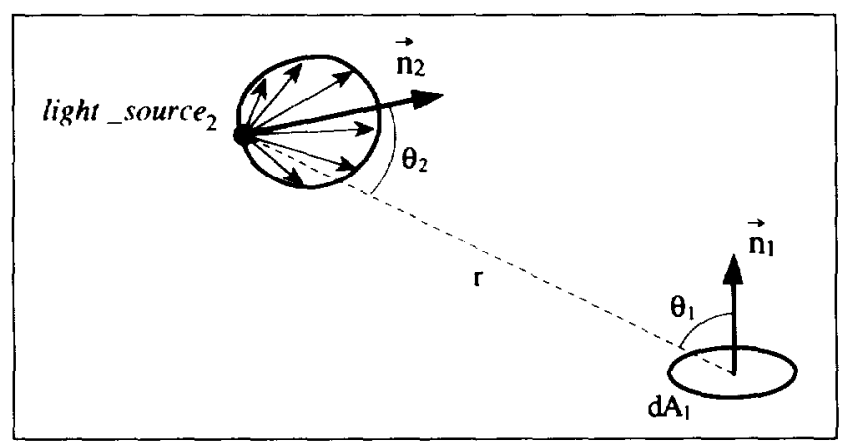

Figure 3: Modeling directional light sources with progressive radiosity.

distribution (Figure 3). The illumination received at vertex 1 from a spot light 2 can be represented by a weighted form-factor based on that light source's emission distribution:

$$
d F_{\text {light_source }-d A_{1}}=d A_{1} \frac{\cos \theta_{1} \cos \theta_{2}}{\pi r^{2}} s\left(\theta_{2}\right)
$$

For directional light sources, a single ray is traced from each vertex to the light for shadow testing. A single ray is sufficient because point sources are used. The amount of energy per unit area received at each vertex is then weighted as shown above. Figure 4 shows a radiosity rendering of several directional light sources.

In this method, the directional light sources initially shoot out their directional energy once, and calculations for any subsequent radiant energy exchange due to secondary reflections can be treated in the standard manner.

\section{Projected Scenery}

\subsection{Overview}

An effective method of creating a scenic background is to project a slide onto a neutral surface. Architectural features, general views, natural objects, cloud formations, and similar objects can be projected on to a backdrop. Projected scenery is fundamentally dif- 


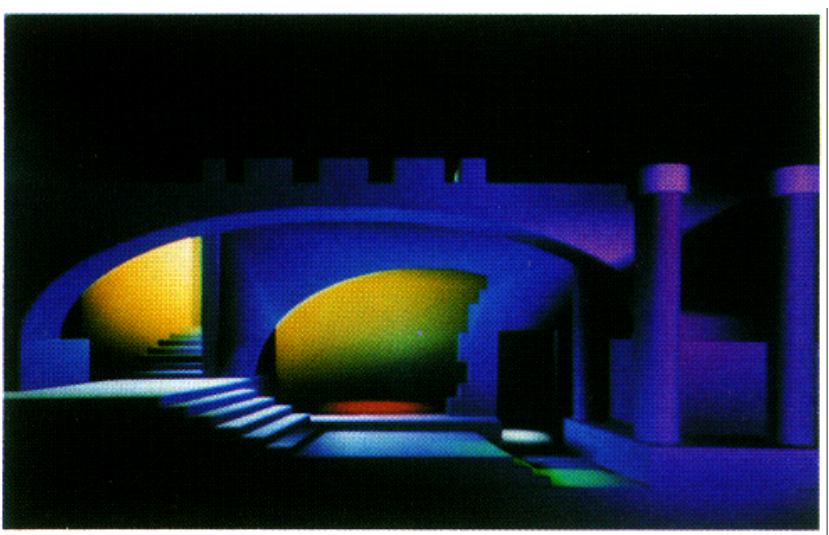

Figure 4: Radiosity rendering of directional light sources.

ferent from built or painted scenery in that it achieves its effects through the use of light. Because color in light is more brilliant than in paint, and has an unlimited value scale by comparison, its use in projection is far more dramatic and eye-catching. Additionally, it can give a greater illusion of size and depth in a setting [27]. In a sense, projection techniques create an imaginary space which extends the bounds of the real space defined by the set geometry. Furthermore, they permit not only rapid changes, but also gradual transitions from one setting to the next, such as from daylight, through sunset to night-all with the same background [11].

Typically, the picture to be projected is painted by hand or photographically produced. Slides for scenic projectors range in size from 5 to 10 inches square. Reduced to its simplest elements, the projection process consists of a light source, the object or slide, the projected image, and the projection surface [18]. There are two types of projection: shadow projection and lens projection. The term lens projection is used to define all projections obtained using one or more lenses; shadow projection is used to describe projections obtained without the use of lenses [26].

\subsection{Simulation of Projected Scenery}

The simulation of background scenery using slide projection techniques is common in opera production, but is new to the field of computer graphics. It is important to derive methods for projection simulation which will maintain the resolution and quality of the original slide, provide soft-focusing according to the optics of the projection system, allow arbitrary geometries for the receiving surfaces, mimic the correct dispersion and attenuation of light, and be computable in tractable amounts of time.

\subsection{A Radiosity Projection Technique}

This section describes an extension to the progressive radiosity algorithm which allows for the projection of scenery. The energy received at the surface of the backdrop from the projection is a function of the emission distribution of the light source, the transmissivity values of the slide, and the orientation and distance of element vertices on the backdrop relative to the projection system. The technique (Figure 5) can be expressed as follows:

1. A two-dimensional array, or "texture map," of values is obtained by scanning photographs or artistic renderings of actual images to be projected.

2. The backdrop/receiving surface is discretized into a series of element vertices, the locations of which are determined based

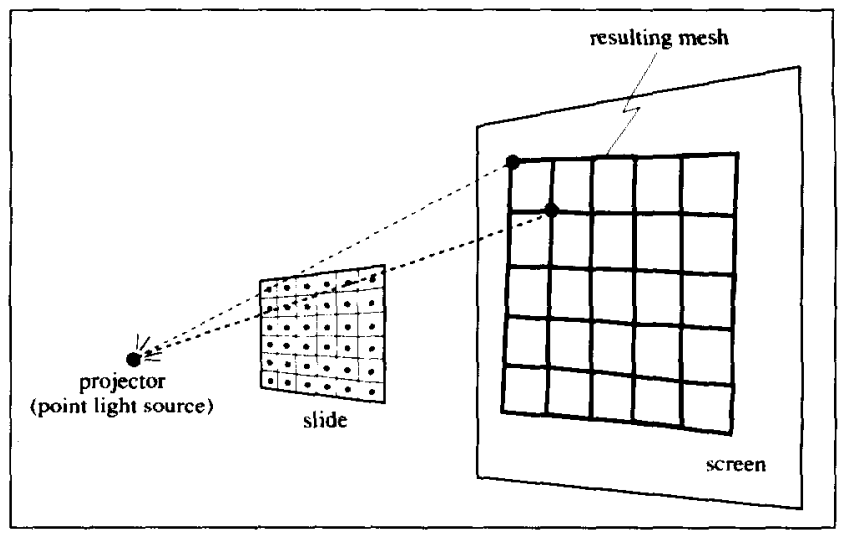

Figure 5: Radiosity Scenic Projection Technique. The backdrop is discretized according to the projected resolution of the slide. The initial radiosity at each element vertex is then based on the emission of the light source, the transmissivity of a point in the slide, and the color of the backdrop.

on the intersection of casting a single ray from the projector through each point of the slide to the backdrop. The resolution of the slide varies according to the desired fuzziness or clarity of the projected image on the backdrop (typically a slide with a resolution between $50 \times 50$ and $100 \times 100=2,500$ to 10,000 element vertices is used).

3. The radiosity of each of these vertices is then based on the color/transmissivity of the relevant point in the slide, the backdrop color, and the emission of the light source in the direction of the element vertex.

The initial radiosity at a vertex on the screen due to the projection (source 2) only is expressed by this modified radiosity equation:

$$
B_{1}=\rho_{1} E_{2} \frac{\cos \theta_{1} \cos \theta_{2}}{\pi r^{2}} s\left(\theta_{2}\right) t_{q}
$$

where,

$E_{2}=$ energy emitted by the point light source,

$t_{q}=$ transmissivity at a point $q$ in the slide.

It should be noted that the effect of a directional light source, $s\left(\theta_{2}\right)$ (projector) is also included in the above equation.

It is also possible, using computer graphics, to model the depth of focus of the projection system. One can "blur" the slide using a filter, sized according to the distance of the projector to the backdrop, so that the entire projection has the same out-of-focus effect. Figure 6 shows a sample projection which was generated using this method.

The radiosity projection technique closely simulates the real projection system in that it yields a view-independent solution with interpolation at the backdrop based on projection characteristics at the maximum stored resolution of the slide. In addition, the backdrop is subdivided according to the projection itself, rather than according to a separate and unrelated element meshing. Finally, the light attenuation of the projector is modeled precisely.

\subsection{A General Solution for the Distortion Problem in Angular Projection}

It is usual to place the projector(s) behind the proscenium, hidden from the view of the audience, and to project downward at an angle to the backdrop. Furthermore, it is common for the backdrop to 


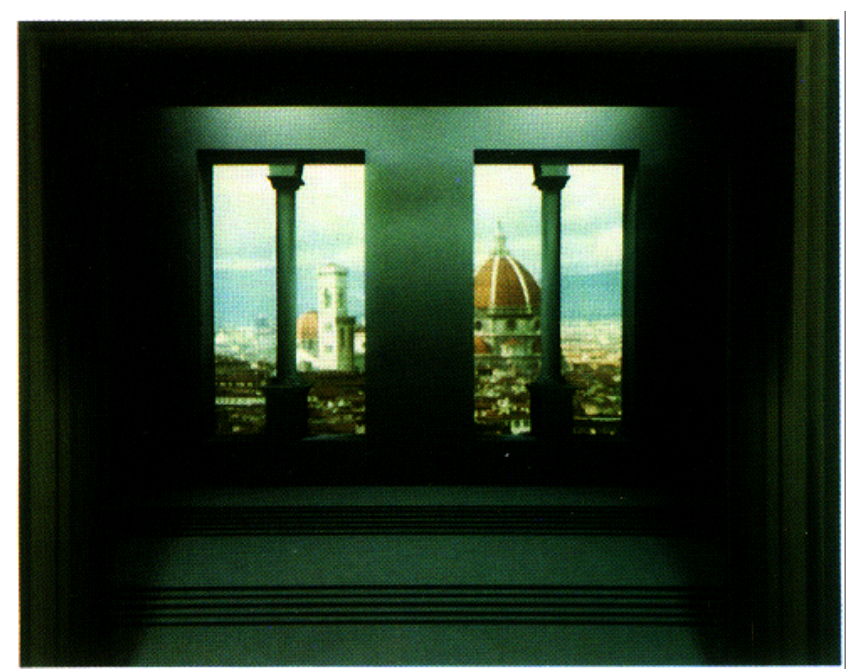

Figure 6: Radiosity Scenic Projection

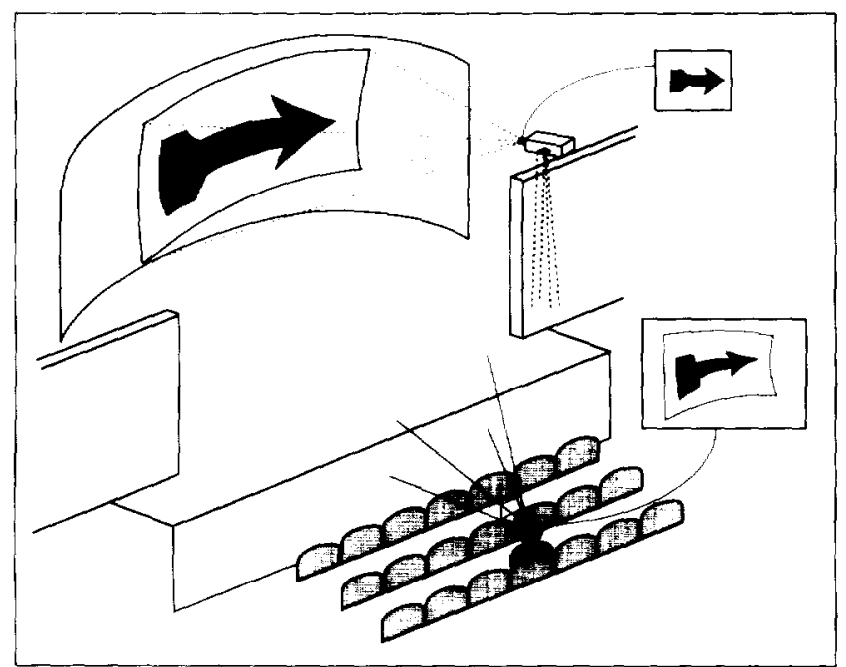

Figure 7: Uncorrected Angular Projection - A square slide in the projector will produce a distorted backdrop image for a viewer.

be non-planar and shaped in some way (e.g. a curved cyclorama). A slide in the projector will therefore produce a distorted backdrop image, which increases in size with the distance from the projector to the backdrop as shown in Figure 7. The problem then is how to predistort a slide such that, when projected and viewed from the position of an "ideal viewer," the backdrop image appears undistorted.

Currently, when a scene is to be projected, the lighting designer waits until the sets are built and in place. Then, a slide (known as a raster) of a regular grid of guide marks is projected from the position where the projector(s) will be located. Next. the resulting projected image (now a distorted grid) is photographed and examined from various viewer positions. Based on the distortion apparent in the projected grid at an ideal viewer location, a predistorted slide is produced using the distorted grid lines as guides. When the slide is projected, the viewer is presented with an undistorted scene. Generally, several iterations of this process are necessary to achieve the desired slide. This trial and error process is time-consuming, labor intensive. and restricted to a given set/projection geometry.

This process can be computer simulated using a digital image warping technique-providing a designer the opportunity to pre- view a projection and produce the distorted slide long before sets are built. The algorithm involves modeling the distortion which would result based on the geometry of a hypothetical projection schema and generating a pre-distorted slide to counteract the distortion.

Image warping is a growing branch of image processing that deals with the geometric transformation of digital images [28]. A geometric transformation is an operation that redefines the spatial relationship between points in an image. The basis of geometric transformations is the mapping of one coordinate system onto another. This mapping is defined by means of a spatial transformation-a mapping function that establishes a spatial correspondence between all points in the input and output images.

Geometric transformations were originally introduced to correct the distortions introduced by remote sensing methods $[2,10]$. This process involved estimating the distortion model, usually by means of a set of reference points. The algorithm presented below differs from these previous techniques in three important respects. First. in this method, one begins with the undistorted image and models the distortion which is introduced. Second, the method is very general in that it does not rely on a particular sensor: it can be used to model any projection system and can account for a projection from any location. Third, this technique involves a two-step approach. because the new value at each point is not merely taken from the mapped coordinates (as is usually the case) but is instead derived from a second mapping (ideal/desired transformation).

\subsubsection{Definitions}

In discussing the problem of an angular projection, it is useful to introduce definitions of the three pertinent coordinate systems. A point on the slide in the projector is referred to as $x_{p}, y_{p} z_{p}$. a point in the projected image on the backdrop as $x_{b} y_{b} z z_{b}$, and a point in the viewer's/spectator's space as $x_{n} y_{1} z_{2}$. The physical slide defines the continuous function of $\left(x_{p} y_{p} z_{p}\right)$ specifying a color at each position.

The ideal siluation would be one in which the projection system would associate points in the projector (slide) and viewer space such that the viewer simply sees a window into the original picture. This operation would define an affine transformation $T$ such that,

$$
\left(x_{n}, y_{1} z_{1}\right)=\left(x_{p} y_{p} z_{p}\right) T
$$

The color (intensity) at some point $x_{c} y_{1} z_{1}$ would then simply depend on the color of the slide at point $\left(x_{p}, y_{p}, z_{\mu}\right)=\left(x_{1} y_{1} z_{1}\right) T^{-1}$.

$$
\left.\left.\operatorname{color}\left(x_{n} y_{r}={ }_{n}\right)=\text { Floriginal_slide }\left(x_{1}, y_{1}={ }_{1}\right) T^{-1}\right)\right]
$$

where the function $F$ describes the effect of light attenuation and illumination (Its exact form does not need to be specified here).

However, in reality, a point on the slide $r_{p}, y_{p} z_{p}$, will be first mapped to the backdrop by the projection, according to some projection transformation, $P$. The resulting point on the backdrop will in turn be transformed to the viewer's space by the usual perspective viewing transformation. $V$. Thus.

$$
\left(x_{b} y_{b}, z_{b}\right)=\left(x_{p} y_{p} z_{p}\right) P^{p}
$$

and,

$$
\left(x_{v} y_{c} z_{l^{\prime}}\right)=\left(x_{b} y_{b} z_{b}\right) V=\left(x_{p} y_{p} z_{p}\right) P V=\left(x_{p} y_{p} z_{p}\right) D
$$

The transformation, $D=P V$, represents the combined mapping from the projection and viewing systems or the distortion, which is ultimately seen by the viewer. There is a parallel between the real distortion, $D$ and the ideal transformation, $T$

The problem is to create a pre-distorted slide such that, after applying the physical transformation $D$ to this slide, the color at each 
point in the viewer space is the same as the color obtained by ideally transforming the original picture using the transformation $T$ :

$$
\begin{gathered}
\forall\left(x_{v} y_{v} z_{v}\right) \quad F\left(\text { original_slide }\left(\left(x_{v} y_{v} z_{v}\right) T^{-1}\right)\right)= \\
F\left(\text { distorted_slide }\left(\left(x_{v} y_{v} z_{v}\right) D^{-1}\right)\right)
\end{gathered}
$$

A simple way of guaranteeing this is to define the distorted slide as:

$$
\begin{aligned}
& \forall\left(x_{p} y_{p} z_{p}\right) \quad \text { distorted_slide }\left(x_{p} y_{p} z_{p}\right)= \\
& \quad \text { original_slide }\left(\left(x_{p} y_{p} z_{p}\right) D T^{-1}\right)
\end{aligned}
$$

\subsubsection{Producing a Pre-distorted Slide}

An algorithm to produce a pre-distorted slide which simulates the actual process used by lighting engineers is presented below. By using Equation (8), the color at every point on the slide is computed. In order to evaluate this equation, a way to compute the distortion $D$ is needed. In general, $D$ is a complex non-linear transformation, and thus it is not practical to evaluate it analytically. The algorithm proposed computes $D$ exactly for a small number of points and uses a linear interpolation to approximate $D$ at all other points. A regular orthogonal grid (the resolution of which can be varied) can be used as a means to quantify the distortion function, $D$. Rays are sent out from the projector at regular intervals (based on the resolution of the grid and the beam angle of the projector) to the projection surface. By transforming the set of grid points from the projector coordi-

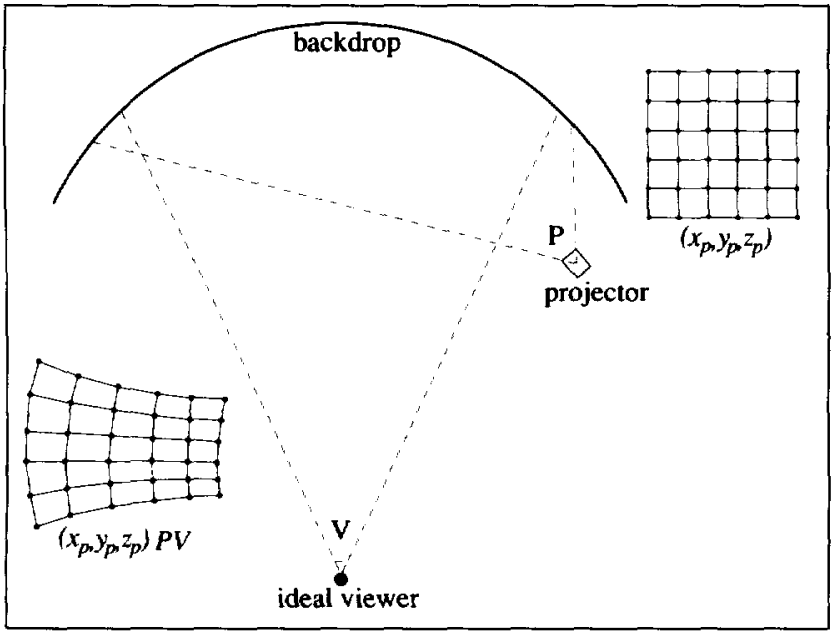

Figure 8: Modeling the distortion in a projection. A regular grid is projected from the projector to the backdrop and mapped to the viewer. The projection system $P$ maps a point from the projector to the screen. The observer's eye-system $V$ maps a point from the screen to the viewer.

nates, $x_{p} y_{p} z_{p}$ to the coordinates on the backdrop/projection surface $x_{b} y_{b} z_{b}$ the function $P$ is simulated. Next, the projected points are transformed into the viewer's coordinates, $x_{v} y_{v} z_{v}$, based on a camera specification which represents the view of an ideal spectator. This transformation represents the function $V$.

The relationship between the original undistorted grid (the set of points $x_{p} y_{p} z_{p}$ ) and the resulting points as seen by the viewer ( $x_{v} y_{v} z_{v}$ ) represents the distortion, $D$, which was produced by the projection (Figure 8 ).

To compute the final slide, the following steps are taken:

First, the virtual, undistorted grid is overlayed onto the slide to be generated as shown in Figure 9 . Next, for each point of the slide, $\left(x_{p} y_{p} z_{p}\right)$ :

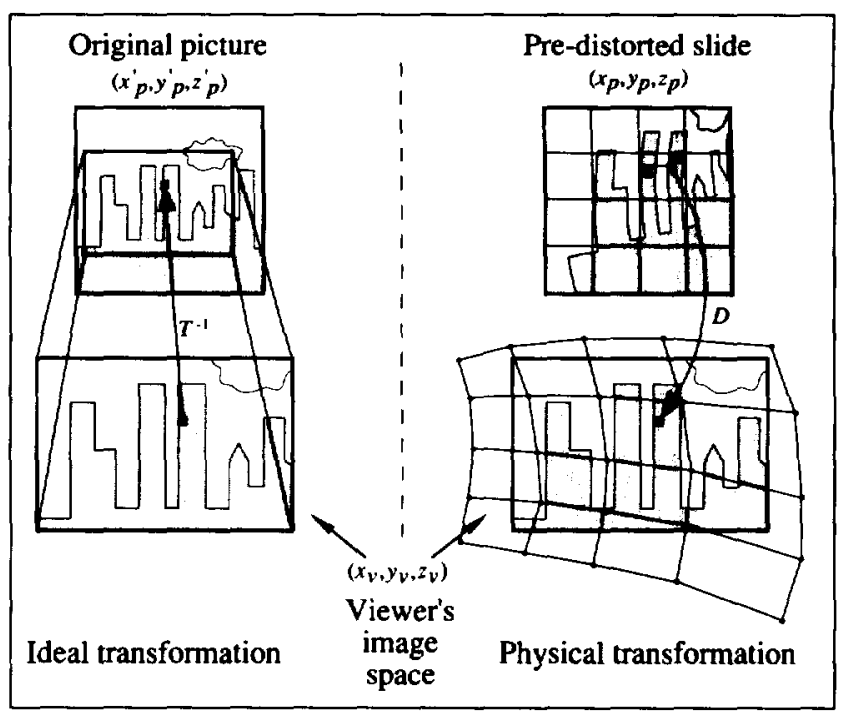

Figure 9: Process for finding the color of a point in the pre-distorted slide.

1. Find which grid cell of the undistorted grid the point is in,

2. Find the coordinates $(u, v)$ of the point within the undistorted grid cell (the local coordinates within the cell),

3. Compute the coordinates of the transformed point $\left(x_{v} y_{v} z_{v}\right)$ in the distorted grid. This computation is accomplished by bilinear interpolation (or a higher order interpolation if necessary) using the four transformed corners of the cell. (At this point the effect of $D$ has been evaluated),

4. Find the point $\left(x_{p}^{\prime} y_{p}^{\prime} z_{p}^{\prime}\right)$ in the original image associated to $x_{v} y_{v} z_{v}$ by the ideal transformation $T^{-1}$. This is a standard windowing operation involving a simple affine transformation,

5. The color of the distorted slide at point $\left(x_{p} y_{p} z_{p}\right)$ is the color of the original slide at point $\left(x_{p}^{\prime} y_{p}^{\prime} z_{p}^{\prime}\right)$.

The result of this procedure is the predistorted slide.

Figure 10 shows a projected image of a New York City skyline onto a curved backdrop along with an illustration of the corrected/pre-distorted slide which was generated using the above method.

\section{Simulation Results}

The geometric information in the opera hall is subdivided into two parts, the geometry of the permanent structure and the geometry and position of the stage sets.

\section{Fixed Geometry - Definition of the Opera Hall}

The geometry of the structure, such as the shell or roof over the stage, as well as the auditorium in general, is fixed. Since the focus of the simulation is only on the stage, a detailed model of the stage portion of the hall has been constructed along with an abstract overall building model. This detailed model includes the proscenium and stage with operable stage lifts as well as the light bridges/gantries where the lights are positioned. The model was 


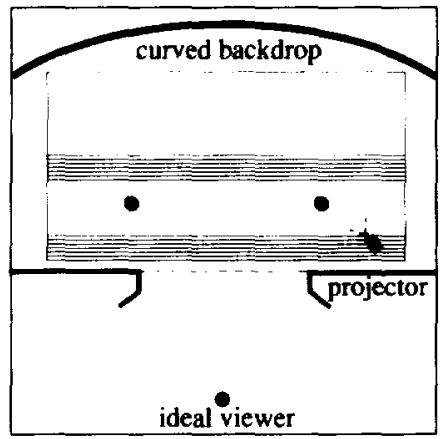

(a)

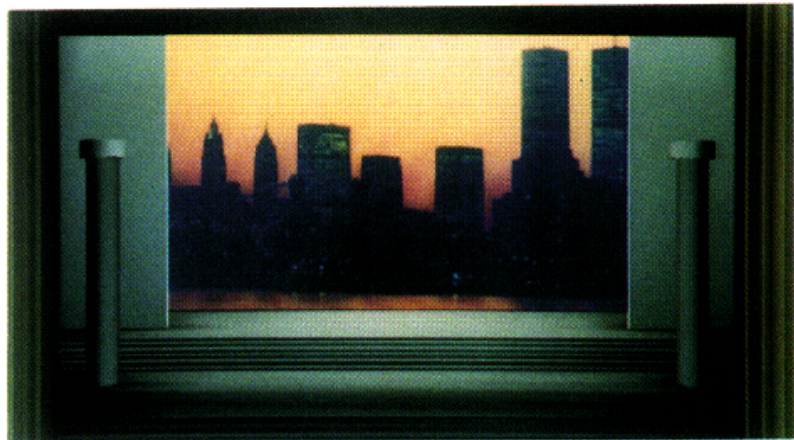

(b)

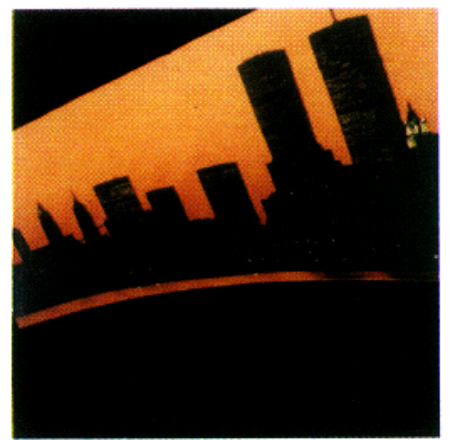

(c)

Figure 10: Radiosity scenic projection of a New York City skyline using a predistorted slide. The projection is from a height of 51.5 feet down at an angle to the curved backdrop. (a) Abstract plan of the environment showing the location of the projector, an ideal viewer, and the shape of the backdrop. (b) Final projected image on the backdrop. (c) Predistorted slide.

made to scale using precise dimensions from drawings and photographs received from the Metropolitan Opera House in New York City.

\section{Variable Geometry - Definition of the Stage Sets \\ Detailed models of the stage sets constitute the second part of the geometric definition. Most of the sets have been defined as extruded contours. Once a set has been modeled, it is possible to position it at any location on the stage and to view it from any position in the hall.}

\section{Examples of Lit Opera Scenes}

To demonstrate the usefulness of the techniques, three full simulations of real productions from the Metropolitan Opera have been generated and are shown in Figures 11,12 and 13. Each of these examples shows the specialized lighting effects-projected scenery, angular projection solutions using pre-distorted slides, and stage lighting-combined with high quality view-independent simulations for practical use in opera design.

Figure 11 shows two views for Günther Schneider-Siemssen's design for the Palazzo on the Grand Canal in Venice from Les Contes d'Hoffmann. The left and right sides of the set are modeled and texture-mapped. The facades in the center background were projected from stage left at a height of 46.5 feet onto a curved backdrop using a pre-distorted slide. Striplights are used to provide an overall wash of light on the set. A series of floodlights were used to simulate moonlight on the facades to the right. A high intensity spotlight produces the effect of the lantern in the doorway.

Figures 12 and 13 show two distinct lighting schemes for Franco Zeffirelli's design of a Parisian garret from La Bohème. A prominent feature in these simulations is the sky, which was projected using two angular projections. The projectors are positioned on a lighting bridge at a height of 53 feet on the left and right sides of the stage. The slides were predistorted to account for the angular projections. A variety of stage lights are positioned on the bridges and used to illuminate the set.

\section{Conclusions}

A set of computer graphics techniques for the design and simulation of opera lighting effects has been presented. The light input, projection, and simulation components give the stage and lighting de- signer a unique opportunity to design, preview, and assess an opera design prior to the construction of full-scale systems. The results of this research clearly demonstrate that the use of computer graphics in theater design holds great promise, particularly since these techniques afford the opportunity for aesthetic evaluations to be made early in the design process and consequently allow many design professionals to work in unison in the preliminary design phase.

Future directions include the use of a higher order interpolation scheme for the projection distortion algorithm, anti-aliasing in the generation of a pre-distorted slide, and the control of the lighting as a function of time.

\section{Acknowledgements}

The authors are extremely grateful to the Hewlett Packard Corporation and the Digital Equipment Corporation for donating the equipment which was used for the simulations and displays. The authors also thank the National Science Foundation for long term support of the research at the Program of Computer Graphics. The Robert James Eidlitz Traveling Fellowship provided funding for a site visit to the Staatsoper, La Scala, and the Metropolitan Opera. Special thanks to Gil Wechsler and Wayne Chouinard at the Metropolitan Opera for providing documentation of real set and lighting designs and for their helpful discussions and input into this research. Mark Shepard modeled the La Bohème environment used in the simulations. Thanks to Kurk Dorsey, Hurf Sheldon, Ben Trumbore, Kevin Novins, Harold Zatz, Ellen French, and Fran Brown for their general assistance. All images were photographed by Emil Ghinger.

\section{References}

[1] Baum, Daniel R., Holly E. Rushmeier, and James M. Winget. "Improving Radiosity Solutions Through the Use of Analytically Determined Form-Factors," Proceedings of SIGGRAPH'89 (Boston, Massachusetts, July 31 - August 4. 1989), in Computer Graphics, 23(3), July 1989, pages 325334.

[2] Bernstein, Ralph. Digital Image Processing of Earth Observation Sensor Data, IBM J. Res. Develop., 1976.

[3] Cayless, M. A. and A. M. Marsden. Lamps and Lighting, Edward Amold, London, 1983. 

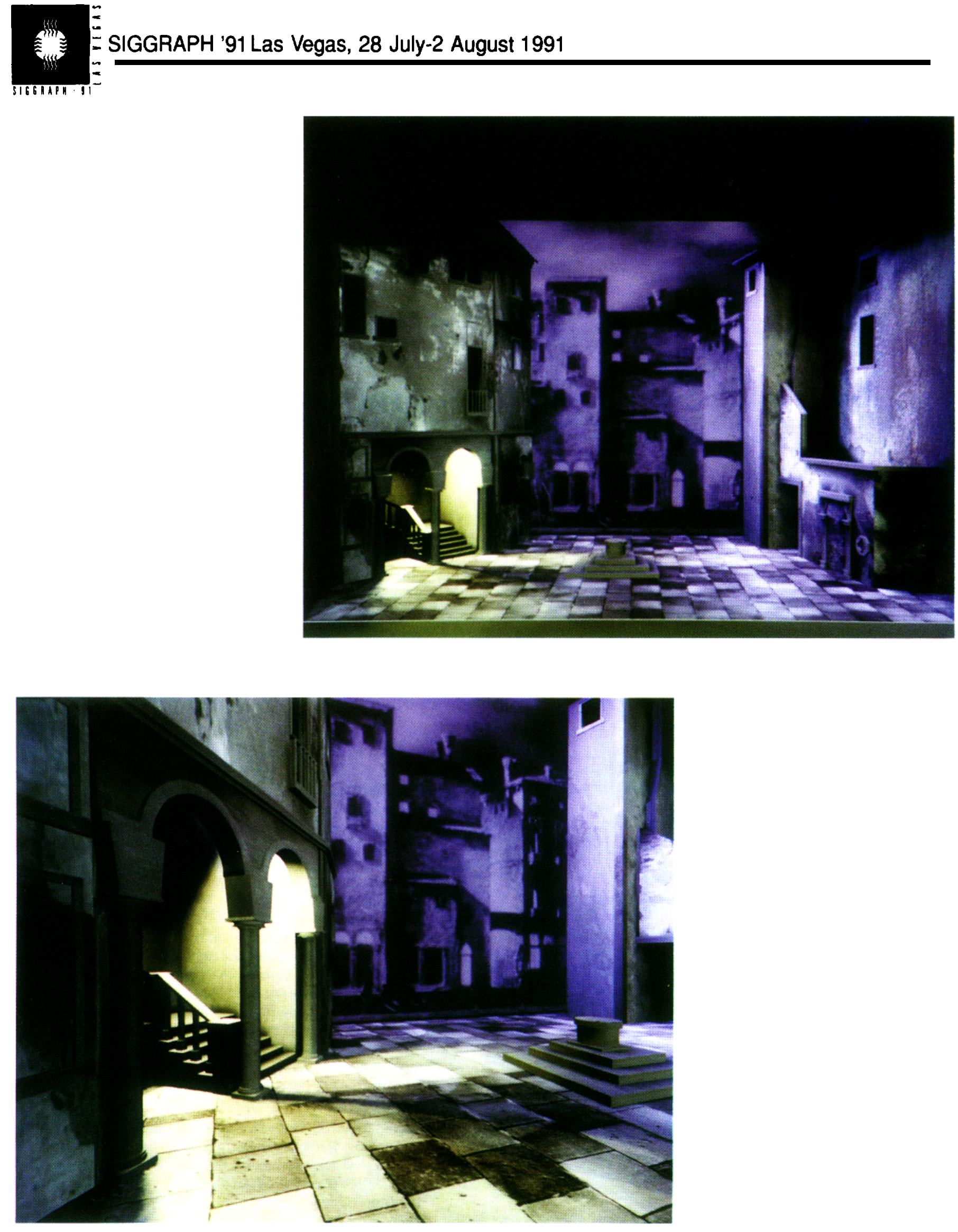

Figure 11: Two views of the Palazzo on the Grand Canal in Venice from Les Contes d'Hoffmann. The left and right sides of the set are modeled. The canal scenery in the center background was projected at an angle onto a curved backdrop using a predistorted slide. A series of floodlights are used to simulate a dim moonlight on the facades to the right. A high intensity spot is used to light the entrance to the left. 


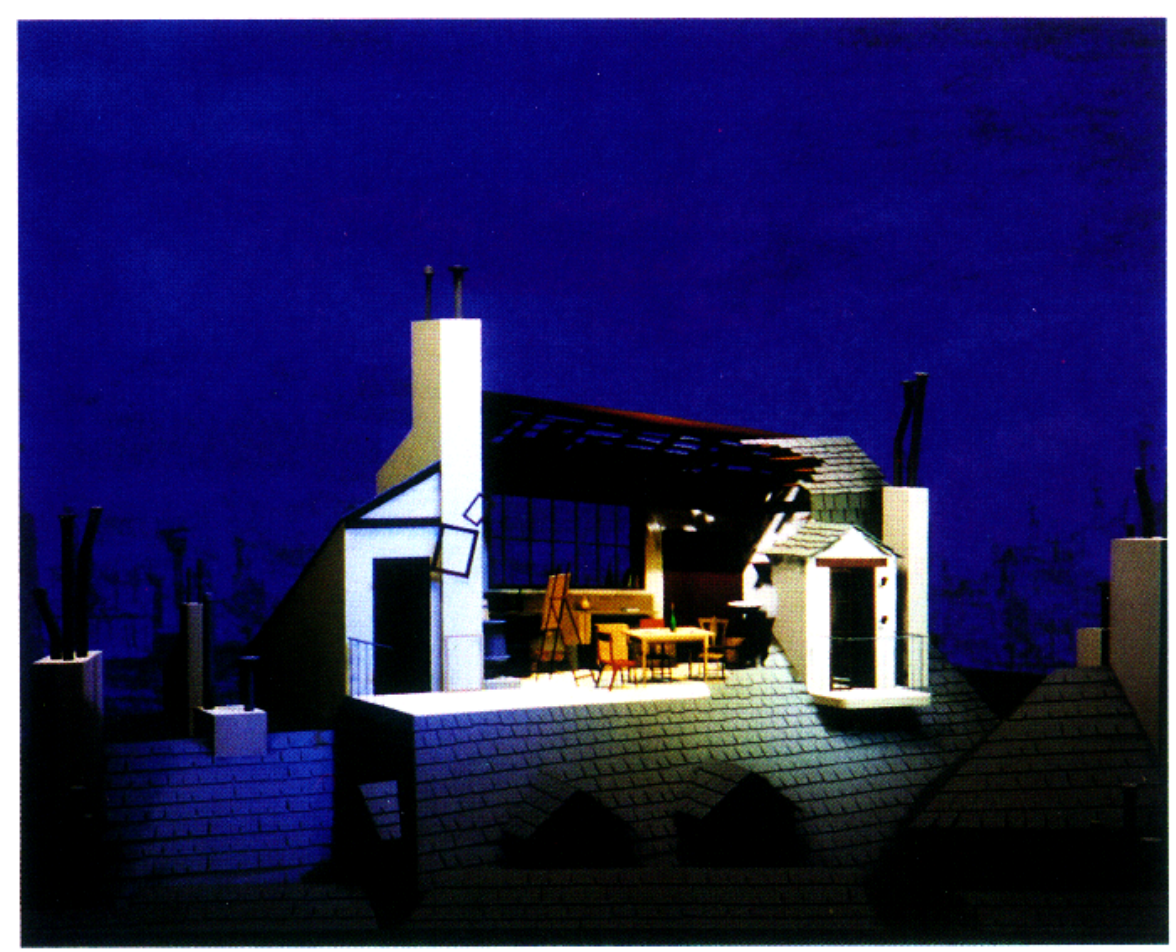

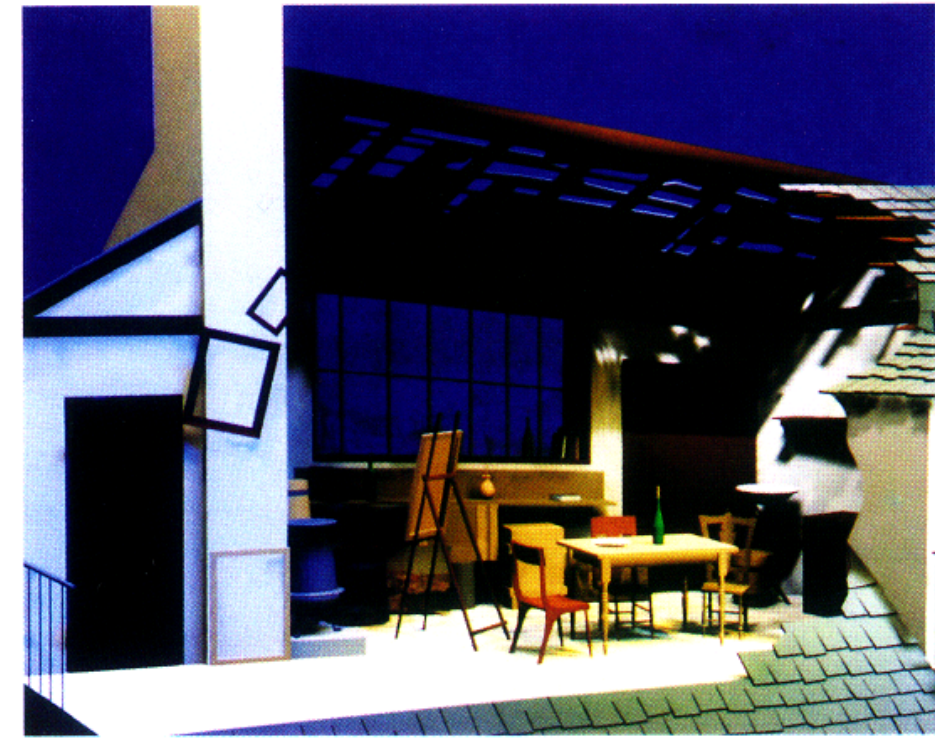

Figure 12 (top and left): Two views of a garret above the rooftops of Paris from La Bohème (evening). The scene contains two angular projections (using predistorted slides) for the sky and a variety of spots and floods. The focus of the lighting is on the entries and the interior of the garret. Figure 13 (right): Alternative lighting scheme for the garret from La Bohème (daytime). The projected slides have been re-colored to obtain the day effect, and additional colored spot lighting emphasizes the center of the set and the right entry.
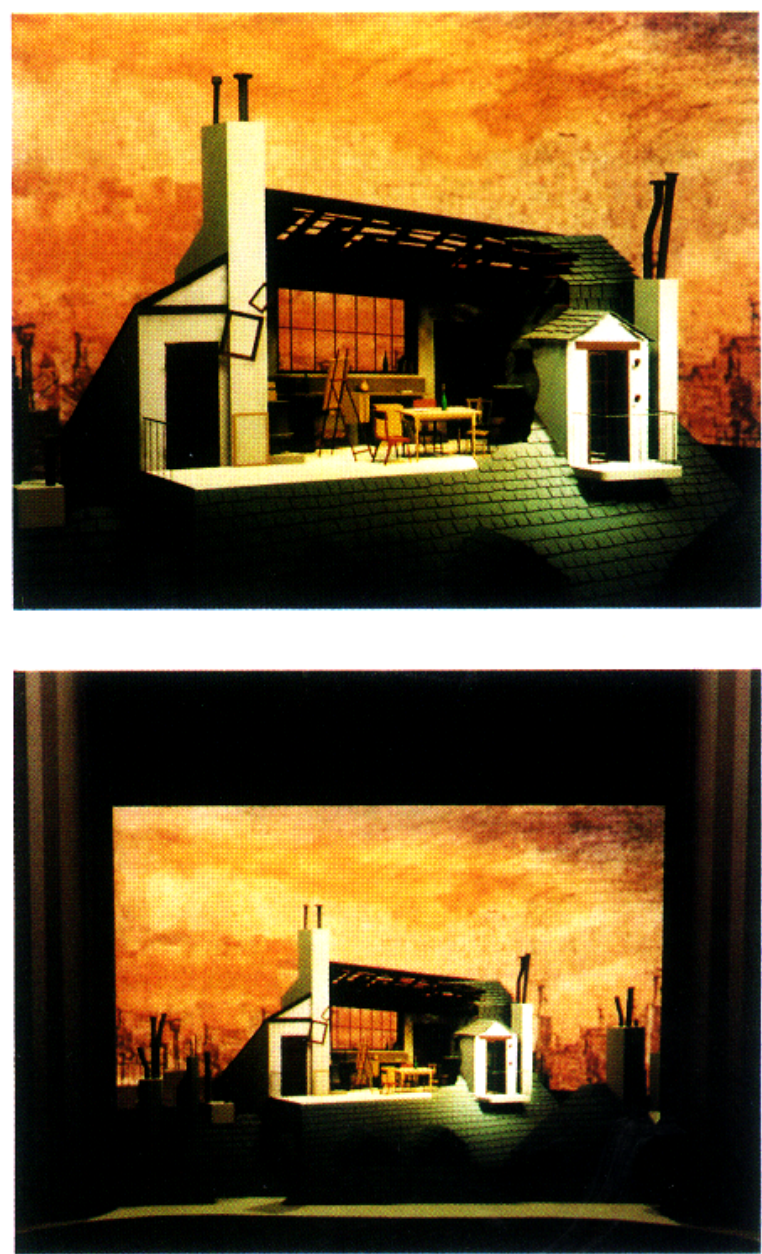
[4] Cohen, Michael F., Shenchang Eric Chen, John R. Wallace, and Donald P. Greenberg. "A Progressive Refinement Approach to Fast Radiosity Image Generation," Proceedings of SIGGRAPH'88 (Atlanta, Georgia, August 1-5, 1988), in Computer Graphics, 22(4), August 1988, pages 75-84.

[5] Cohen, Michael F. and Donald P. Greenberg. "A Radiosity Solution for Complex Environments," Proceedings of SIGGRAPH'85 (San Francisco, California, July 22-26, 1985), in Computer Graphics, 19(3), July 1985, pages 31-40.

[6] Cohen, Michael F., Donald P. Greenberg, David S. Immel, and Philip J. Brock. "An Efficient Radiosity Approach for Realistic Image Synthesis," IEEE Computer Graphics and Applications, 6(3), March 1986, pages 26-35.

[7] Dorsey, Julie O'B. Computer Graphics for the Design and Visualization of Opera Lighting Effects, Master's thesis, Program of Computer Graphics, Comell University, Ithaca, NY 14853, January 1990.

[8] Gilette, Michael J. Designing With Light, Mayfield Publishing Company, Palo Alto, 1978.

[9] Goral, Cindy M., Kenneth E. Torrance, Donald P. Greenberg, and Bennett Battaile. "Modeling the Interaction of Light Between Diffuse Surfaces," Proceedings of SIGGRAPH'84 (Minneapolis, Minnesota, July 23-27, 1984), in Computer Graphics, 18(3), July 1984, pages 213-222.

[10] Haralick, Robert M. "Automatic Remote Sensor Image Processing," Topics in Applied Physics, 11, 1976, pages 5-63.

[11] Hartmann, Rudolf. Opera, William Morrow and Company, New York, N.Y., 1977.

[12] Heckbert, Paul S. Fundamentals of Texture Mapping and Image Warping, Master's thesis, Department of EECS, UC Berkeley, Berkeley, CA 94720, June 1989.

[13] Kaufman, John E. IES Lighting Handbook - Application Volume, Illuminating Engineering Society of North America. New York, 1987.

[14] Max, Nelson L. "Computer Graphics Distortion for IMAX and OMNIMAX Projection," in Proceedings of Nicograph'83, 1983.

[15] Nishita, Tomoyuki, Yasuhiro Miyawaki, and Eihachiro Nakamae. "A Shading Model for Atmospheric Scattering Considering Luminous Intensity Distribution of Light Sources," Proceedings of SIGGRAPH'87 (Anaheim, California, July 27-31, 1987), in Computer Graphics, 21(4), July 1987, pages 303-310.

[16] Nishita, Tomoyuki and Eihachiro Nakamae. "Continuous Tone Representation of Three-Dimensional Objects Taking Account of Shadows and Interreflections," Proceedings of SIGGRAPH'85 (San Francisco, California, July 22-26, 1985), in Computer Graphics, 19(3), July 1985, pages 23-30.

[17] Nishita, Tomoyuki, Isao Okamura, and Eihachiro Nakamae. "Shading Models for Point and Linear Sources," ACM Transactions on Graphics, 4(2), April 1985, pages 124-146.

[18] Parker, W. Oren and Harvey K. Smith. Scene Design and Stage Lighting, Holt, Rinehart and Winston, Inc., New York, 1979.
[19] Pilbrow, Richard. Stage Lighting, Von Nostrand Reinhold Company, New York, 1979.

[20] Sellman, Hunton D. and Merrill Lessley. Essentials of Stage Lighting, Prentice-Hall, Inc, Englewood Cliffs, 1982.

[21] Siegel, Robert and John R. Howell. Thermal Radiation Heat Transfer, Hemisphere Publishing Corp., Washington DC., 1981.

[22] Sillion, François and Claude Puech. "A General Two-Pass Method Integrating Specular and Diffuse Reflection," Proceedings of SIGGRAPH'89 (Boston, Massachusetts, July 31 - August 4, 1989), in Computer Graphics, 23(3), July 1989, pages 335-344.

[23] Verbeck, Channing P. and Donald P. Greenberg. "A Comprehensive Light-Source Description for Computer Graphics," IEEE Computer Graphics and Applications, 4(7), July 1984, pages 66-75.

[24] Wallace, John R., Kells A. Elmquist, and Eric A. Haines. "A Ray Tracing Algorithm for Progressive Radiosity," Proceedings of SIGGRAPH'89 (Boston, Massachusetts, July 31 - August 4, 1989), in Computer Graphics, 23(3), July 1989, pages 315-324.

[25] Warn, David R. "Lighting Controls for Synthetic Images," Proceedings of SIGGRAPH'83 (Detroit, Michigan, July 25$29,1983)$, in Computer Graphics, 17(3), July 1983, pages 1321.

[26] Watson, Lee. Lighting Design Handbook, McGraw-Hill, New York, 1990.

[27] Wilfred, Thomas. Projected Scenery - A Technical Manual, The Drama Book Shop, New York, 1965.

[28] Wolberg, George. Digital Image Warping, IEEE Computer Society Press., Los Alamitos, CA, 1990

\section{Appendix A - Beam and Field Angles}

The beam angle is the central cone of light emitted from an instrument (Figure 14). The limit of the beam angle is usually defined as

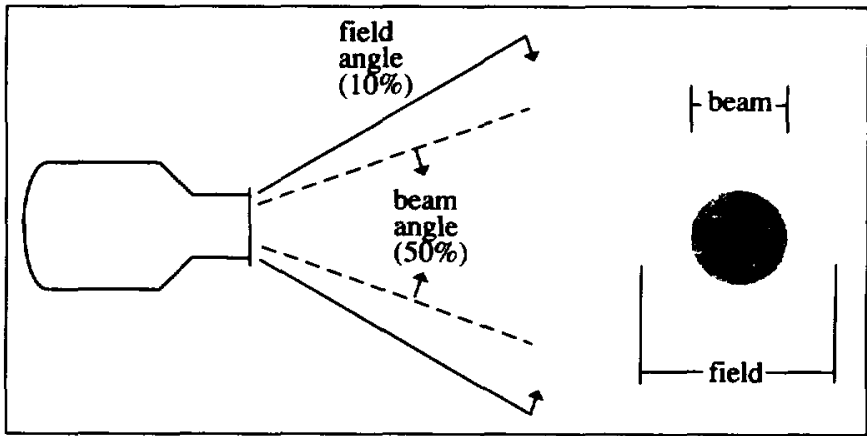

Figure 14: Relationship between the beam and field angles of a lighting instrument.

that point where the light diminishes to 50 percent of its intensity when compared with the center of the beam. The field angle is described as that point where the light diminishes to 10 percent of the output of the center of the beam. 